\title{
Traffic conflicts analyses for $2+1$ road sections
}

\author{
Salvatore Cafiso ${ }^{1}$, Carmelo D'Agostino ${ }^{1}$, Radosław Bąk ${ }^{2}$, and Mariusz Kieće,* \\ ${ }^{1}$ University of Catania, Via Santa Sofia 64 . Catania, Italy \\ ${ }^{2}$ Cracow University of Technology, Warszawska 24, 31-155 Krakow, Poland
}

\begin{abstract}
The additional passing lanes and $2+1$ roads improve significant road safety. Studies indicate sections with additional passing lanes (relief or alternately), which may cause reduction in the number of accidents by $50 \%$. However, how geometric design affects the safety performance of such sections is not in depth investigated. Previous studies are carried out with two approaches, i.e. the most often, based on analysis of observed crashes and more rarely by using microsimulation study. In the case of microsimulation research, traffic conflict theory can be applied as a surrogate measure of safety. One of the main problem in simulated conflicts study is the validation of simulation results against real world conditions. The aim of the paper is to assess the reliability of traffic conflict measures obtained by microsimulation against real world observation. Conflicts were detected and classified from video recording and analysis of vehicle trajectories in the merging area on $2+1$ roads in Poland. Conducted studies focus only on lane changing conflicts, locations and TTCs values of observed conflicts between vehicles were primarily identified. Observed conflicts are than compared with microsimulated one, to assess if there is a correlation in the two.
\end{abstract}

\section{Introduction}

Improving safety and operational performance of new highway design solutions from observational data is a reactive approach that requires a suitable sample of sites and a historical evaluation. As far as the safety performance is concerned, SPFs and CMFs are statistically-based prediction methods which require significant efforts in crash data collection and long periods to observe crash occurrence. Traffic conflict studies can mitigate this issue using a short time survey to count the number and severity of traffic conflicts which are assumed as surrogate measures of safety. Unfortunately, they are again field-based studies which can be carried out only after the implementation of the treatment. Moreover, international transferability of existing studies is another issue which limits the predictability of future performances basing on literature case studies [1-3]. That is the case of the evaluation of safety performance of new design schemes or treatment typologies such as the design of $2+1$ lanes in Poland, objective of the present research work. Nowadays, microsimulation is well established tool for analyzing the operational effects of new or complex geometric schemes [4,5]. More recently, studies [6] are attempting to use

\footnotetext{
* Corresponding author: $\underline{\text { mkiec@.pk.edu.pl }}$
} 
microsimulation vehicle trajectories to classify traffic conflicts, as well. Specifically, traffic conflicts can be detected through the Surrogate Safety Assessment Model software (SSAM) [7] where vehicle trajectories obtained from microsimulation software (e.g. VISSIM) are post-processed. The application of microsimulated conflicts is considered as one of the methods based on surrogate measures which allows to estimate the number of conflicts for different road and traffic configurations [8-12]. Such an approach is much more popular in the analysis of intersections and, although simulation modeling for road sections with additional lanes is described in literature, these limited studies mainly discuss the modeling in the context of the evaluation of traffic performance $[13,14]$. Moreover, despite the wellknown potentialities of these software tools, care has to be taken regarding the appropriateness of the microsimulation in reproducing the real word number and severity of traffic conflicts. Therefore, calibration of models and validation of results should be carefully considered. In this framework the present research work deals with alternate passing lanes which differ from the traditional $2+1$ scheme due to a limited number of segments with the added lane (usually only one per direction). In Poland the approach is to build alternate $2+1$ passing lane, and to admit long two lane sections between $2+1$ road section. The reasons of the application of such design in Poland include: the high density of intersections and sub-urban area and budget constraints for a comprehensive reconstruction of the entire road network. Short passing sections can limit the main functions of longer $2+1$ sections, i.e. the ability to disperse traffic platoons and it may lead to hazardous manoeuvres like aggressive lane changing and increase in number of conflicts in the critical merging area at the end of the $2+1$ section. Starting from microsimulation scenarios calibrated on operational parameters by Authors [15], the present research work deals with the validation of the simulated conflicts against observed conflicts. At this stage of the research the paper focuses mainly on the video recording and evaluation to get observed conflicts from digital image software analysis, vehicle tracking and algorithms to detect conflicts in real world $2+1$ sections. The observations were conducted in 5 locations with different geometric design and traffic flows. Microsimulation in VISSIM [16] environment are carried out and SSAM conflicts are identified and counted, as well. Finally, to assess a correlation study between observed and simulated number of conflicts is presented as first step to validate microsimulation results.

\section{Methodology}

Comparison between observed and simulated conflicts requires using microsimulation model and conducting empirical research. Microsimulation model was developed with the PTV VISSIM software. In order to calculate conflicts based on trajectories obtained from microsimulations SSAM software was used. Observed conflicts were calculated based on video observations and analysis of digital frames to detect location of vehicles frame by frame.

\subsection{Simulated conflicts}

The development of the simulation model and calculation of simulated conflicts are described in detail in the $[11,15]$. To develop microsimulation model the empirical data was collected during field observations at 5 test sites on $2+1$ road sections in Poland. The field observations were conducted during the day, in good weather conditions for a minimum of 4-5 hours including peak hour periods. The lengths of the section with the additional lane ranged from $550 \mathrm{~m}$ to $1000 \mathrm{~m}$ while the traffic volume (calculated within 5-minute intervals) fells within the range of 240 to $1,116 \mathrm{veh} /$ hour/dir. All sections were located on level terrain with vertical grade alignment lower than $3 \%$. The data was collected with the 
help of pneumatic sensor traffic recorders and ANPR cameras (Automatic Number Plate Recognition). The field study documented driver behavior and traffic operation at the beginning and at the end of the sections. Microsimulation model was calibrated and validated. The aim of the calibration was to obtain a reliable share of platooning vehicles at the entry and exit of the passing lane in simulation environment. The calibration has been performed on a section with $1000 \mathrm{~m}$ of passing lane length. In order to perform the calibration, the desired speed distribution for vehicles on the passing lane has been chosen as a variable in the software by changing it from the default values to make the results able to match the observed one. The share of overtaking vehicles has been selected as validation parameter. The model validation has been performed on the data of the $800 \mathrm{~m}$ passing lane. For both calibration $(1000 \mathrm{~m})$ and validation $(800 \mathrm{~m})$ sections, MAPE of share of overtaking vehicles resulted lower than $10 \%[17,18]$. Traffic conflicts were estimated by SSAM software using microsimulation trajectory outputs of VISSIM after calibration and validation. The analysis focused only to lane-changing conflicts. The conflicts were estimated for Time to Collision (TTC), for passing relief lane lengths varying from $500 \mathrm{~m}$ to $1200 \mathrm{~m}$ with steps of $100 \mathrm{~m}$. All the simulations reported above, considered a length of the merging area equal to $100 \mathrm{~m}$.

\subsection{Observed conflicts}

Observed conflicts were gathered during a survey campaign in different $2+1$ segments in Poland in 3 locations in various conditions of geometry, traffic, share of heavy vehicles and speed. Particularly the areas under investigation are all located in Poland in the territory of the Municipality of Piaski, Zyrardow and Krasnik (Figure 1). When surveys were performed there was not physical separation in the median [19].
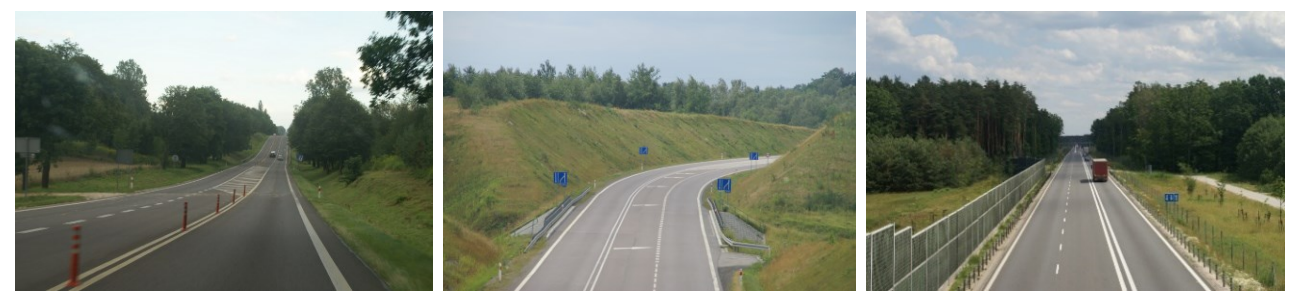

Fig. 1. Piaski (a), Krasnik (b) and Zyrardow (c).

For each test site the location of cameras was chosen to ensures an excellent view of the merging area and of vehicle paths. Surveys were usually carried out during peak traffic hour (from 12:00 to 14:00) during week days (from Tuesday to Thursday). Generally, the best position is on the right side of the merging area at a distance from the start of the merging area of about $90-100 \mathrm{~m}$. It is preferable that the observer and all the equipment should be not visible for not affecting the driver behavior. Another factor that must be considered, is that if the cameras are located at the street level, video cannot record clearly vehicles traveling on the inner lanes (above all when heavy vehicle are present in the video frames), so it is preferable that they are positioned at a height from road level of at least $4 / 5$ $\mathrm{m}$. Table 1 reports the geometric characteristics of the additional lane selected for the video survey. Each $2+1$ segment was surveyed for at least 2 hours (15 minutes was used as warm up time) with a high resolution camera placed at a distance of about $100 \mathrm{~m}$ to the starting of the merging area. This position was chosen based on the microsimulation results, which 
assess that mainly the conflicts are located in that part and only a negligible number of them are in other zone of the $2+1$ segments [11].

Table 1. Summary of $2+1$ Sections Characteristics.*

\begin{tabular}{|c|c|c|c|}
\hline \multirow{2}{*}{ Roadway } & $\begin{array}{c}\text { Additional lanes length } \\
{[\mathbf{m}]}\end{array}$ & $\begin{array}{c}\text { non-conflicting } \\
\text { changeover length }[\mathbf{m}]\end{array}$ & $\begin{array}{c}\text { conflicting changeover } \\
\text { length }[\mathbf{m}]\end{array}$ \\
\hline \multirow{2}{*}{ Zyrardow } & 1000 & 60 & 160 \\
\cline { 2 - 4 } & 800 & 60 & 160 \\
\hline \multirow{2}{*}{ Krasnik } & 1100 & non-typical changeover & non-typical changeover \\
\cline { 2 - 4 } & 1500 & non-typical changeover & non-typical changeover \\
\hline \multirow{2}{*}{ Piaski } & 1170 & 130 & 150 \\
\cline { 2 - 4 } & 850 & 130 & 130 \\
\hline
\end{tabular}

*All the section has grades in the $\pm 2 \%$ range.

As mentioned earlier the data gathering was made through video recordings in the different sections under investigation. The area analyzed in the video recording was a lengths ranging from 85 to 100 meters from conflicting changeover starting point and a width of 10.50 meters (Figure 2). Before starting the video recording, based on the location some fixed points were taken as reference and made them visible on the frame. The distance between those fixed points were than measured to calibrate the software.

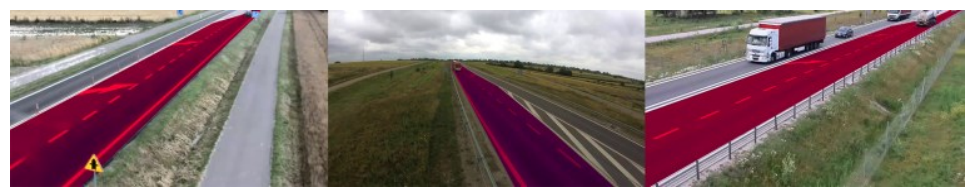

Fig. 2. Piaski (a), Krasnik (b) and Zyrardow (c).

\section{Microsiumulation model validation with observed conflicts}

Microsimulation model are calibrated using observed data, related to traffic operation parameter. As reported elsewhere, simulated conflicts are a surrogate measure of safety $[11,15]$. By comparing observed conflicts with simulated one, it is expected to validate simulation models even for traffic safety analysis.

\subsection{Data treatment}

Based on empirical research 58 conflicts were identified in the 10 hours of video recording (Table 2) and used in the comparison with simulated conflicts. Looking at Table 2, it is possible to assess a great share of heavy vehicle, which go over $50 \%$ in some cases. Each vehicle traveling in the passing lane with lane-changing conflicts, was at first identified in the 10 hours of video recording and then the set of frames was analyzed separately by using an open source software "Sputnik" [20] for vehicles tracking and positioning. Sputnik output are the positions in the different time frames (50 frame per second) of each vehicle $(\mathrm{x}, \mathrm{y})$ in the reference system.

The original output of vehicle trajectories showed considerable noise due to detection and tracking precision. Therefore, a filter should be applied before further elaboration of data. A simple moving average filter resulted enough effective for smoothing the irregularities in the trajectories and speed profiles of vehicles. Other algorithms were applied to identified the conflict point and Time to Collision. The flow chart of the overall procedure is reported in Figure 3. 
Table 2. Summary of 2+1 Sections Characteristics and Number of Observed Conflicts.

\begin{tabular}{|c|c|c|c|c|}
\hline \multirow{2}{*}{ Roadway } & $\begin{array}{c}\text { 1 hour } \\
\text { interval }\end{array}$ & $\begin{array}{c}\text { Traffic volume ALL } \\
\text { [veh/hour] }\end{array}$ & $\begin{array}{c}\text { Traffic volume - Heavy } \\
\text { vehicles [veh/hour] }\end{array}$ & $\begin{array}{c}\text { number of } \\
\text { conflicts }\end{array}$ \\
\hline \multirow{2}{*}{ Zyrardow 1 } & 1 & 471 & 274 & 8 \\
\cline { 2 - 5 } & 2 & 518 & 299 & 8 \\
\hline \multirow{2}{*}{ Krasnik 1 } & 1 & 201 & 84 & 7 \\
\cline { 2 - 5 } & 2 & 219 & 94 & 0 \\
\hline \multirow{2}{*}{ Krasnik 2 } & 1 & 198 & 84 & 0 \\
\cline { 2 - 5 } & 2 & 216 & 94 & 6 \\
\hline \multirow{2}{*}{ Piaski 1 } & 1 & 407 & 99 & 2 \\
\hline \multirow{2}{*}{ Piaski 2 } & 2 & 610 & 125 & 2 \\
\hline \multirow{2}{*}{ TOTAL } & 1 & 345 & 92 & $\mathbf{5 8}$ \\
\hline
\end{tabular}

The original output of vehicle trajectories showed considerable noise due to detection and tracking precision. Therefore, a filter should be applied before further elaboration of data. A simple moving average filter resulted enough effective for smoothing the irregularities in the trajectories and speed profiles of vehicles. Other algorithms were applied to identified the conflict point and Time to Collision. The flow chart of the overall procedure is reported in Figure 3.

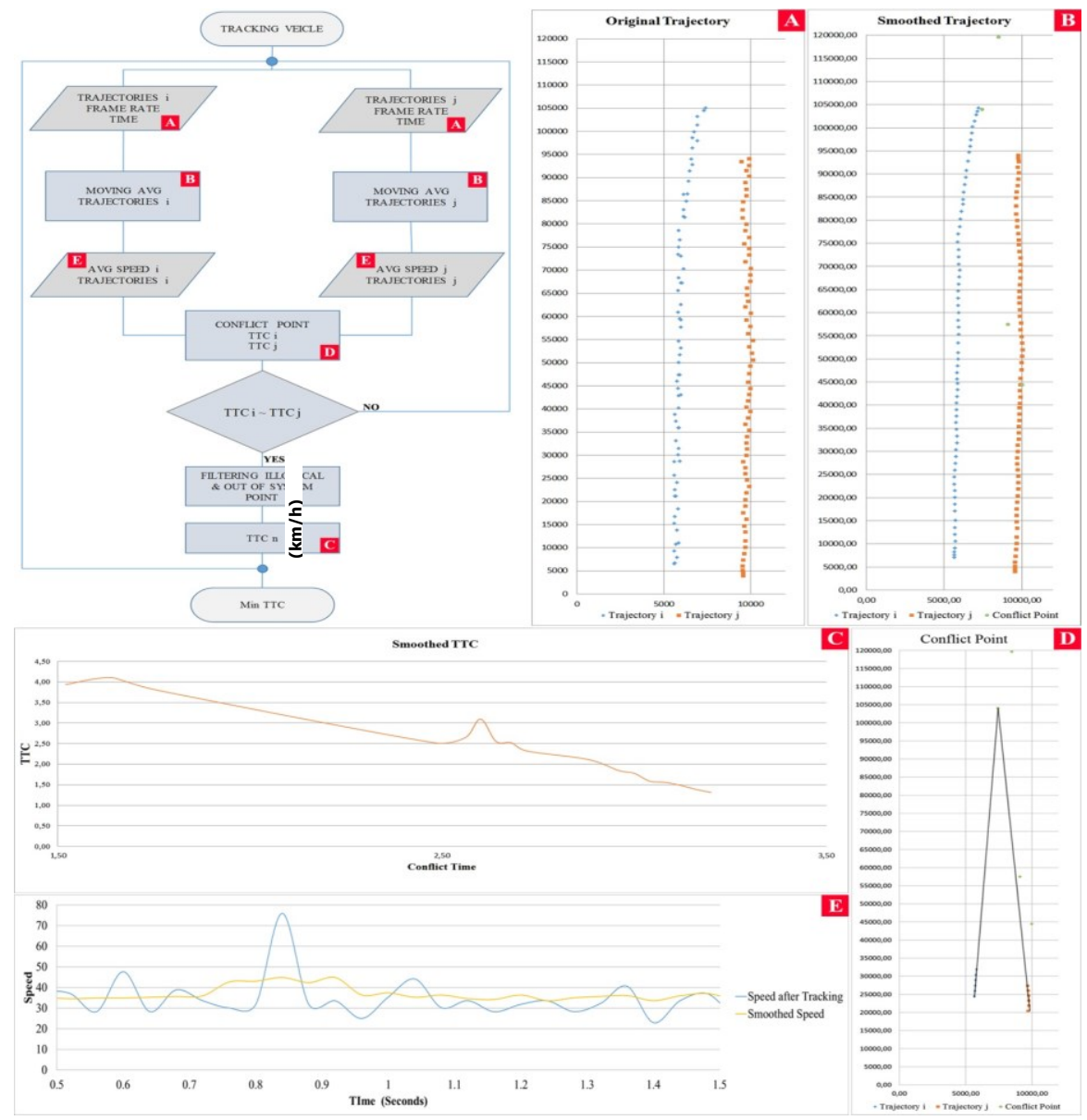

Fig. 3. Algorithm for the post elaboration of conflicts and its features (unit of trajectories and conflict point is millimetre) and conflict map example. 
For each conflict two vehicles are tracked, one in the regular lane and the other in the passing lane. After the smoothing of the trajectories (Figures A and B), for each vehicle the instantaneous trajectory is extrapolated and the conflict point identified by the intersection of the two trajectories (Figure D). The time to collision of each vehicle is calculated as ratio between the distance to the conflict point and the speed (Figure E). If the time to collision of the two vehicles (TTCi, TTCj) is equal with an accepted tolerance (e.g. 0.2 seconds) that condition is classified as a conflict with TTC and the next positions of the two vehicles is analyzed. After the end of the conflict evaluation, when the vehicles exit from the video window, the conflict with the minimum TTC (Figure C) is recorded for further evaluations. Based on the conflict maps defined as the position of the conflict point at the minimum TTC, it is possible to identify that the conflict are more concentrated at the end of the merging lane and mainly located on the regular lane, as expected.

\subsection{Comparison between observed and simulated conflicts}

With the aims to compare observed and simulated conflicts, a regression analysis was performed on the number of simulated conflicts aggregated in 1 hour for different traffic flows and geometric characteristics. SSAM conflicts were selected in a range of TTC from 1 to 5 seconds from the microsimulation models. As conflict occurrence and repetition follow the same probability distribution of crashes, like as crash number [21,22], it is possible to assume a negative binomial (NB) distribution for traffic conflict frequency. Therefore, a Generalized Linear Modeling with NB distribution [11] is a suitable tool for regression analysis. The regression is needed to estimate conflicts in the same condition of traffic and section length of the $2+1$ sections observed in the real condition. Particularly, based on observed $2+1$ section data (Tables 1,2), 80 hours of simulated conflicts were considered with a length varying from 500 to $1200 \mathrm{~m}$ with steps of $100 \mathrm{~m}$ and traffic flows from 300 to $1200 \mathrm{veh} /$ hour/dir with a step of $100 \mathrm{veh} / \mathrm{hour} / \mathrm{dir}$. Each simulation was run for 5 hours after a $30 \mathrm{~min}$ of warm up time. The chosen model form is the following:

$$
\frac{\text { Conflicts }}{\text { hour }}=e^{\alpha} \cdot Q^{\beta} \cdot L
$$

where: $L$ : length of additional passing lane [m]; $Q$ : traffic volume [veh/hour/dir] $\alpha, \beta$ : regression coefficients. After model calibration, both the model and the independent variables resulted significant and the regression coefficients maximizing the log-likelihood function are reported in Table 3 From Table 3, the Pearson Chi-square statistic is small enough to assess a fair goodness of fitting (gof) of the regression model to the data. Real world conflicts identified by the video analysis were compared with the simulated ones in the same geometric and traffic conditions.

Table 3. Regression Parameters and gof for Equation 1 ad represetation.

\begin{tabular}{|c|c|c|c|}
\hline & Coeff. & Stdev & P-Value \\
\hline (Intercept) $\boldsymbol{\alpha}$ & -30.7971 & 1.1707 & $<.0001$ \\
\hline (Traffic) $\boldsymbol{\beta}$ & 3.9886 & 0.1710 & $<.0001$ \\
\hline Dispersion parameter k & 0.0542 & - & - \\
\hline Scaled Deviance (SC/DF*) & \multicolumn{3}{|c|}{$68.71(0.88 \mathrm{DF}=78)$} \\
\hline $\begin{array}{c}\text { Pearson Chi-square } \\
\text { (Chi-square/DF) }\end{array}$ & \multicolumn{3}{|c|}{78.0620} \\
$(1.0008 \mathrm{DF}=78)$ \\
\hline
\end{tabular}

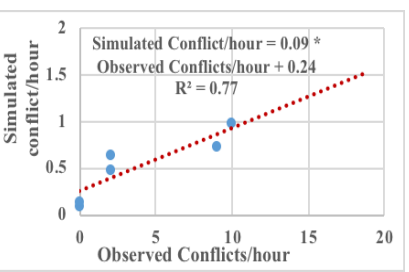

A straight linear correlation was observed between observed and simulated number of conflicts with a ratio of 10 real conflicts for each simulated one (Table 3 ). On the other hand, simulation gives always conflict also when in the very low traffic conditions no one is observed. The regression coefficient was very high with a value of $\mathrm{R}^{2}$ equal to 0.8 . Those 
results show that at low traffic volumes longer observation periods are need. Basing also on the simulation results, at least 5 hours of observations is a minimum time for acquiring enough data with traffic flows of 200 vehicles per hour. Regression results shown a general goodness of fit of the regression analysis performed on simulated conflicts applied to estimate the number of observed conflicts. In the early stage of the investigation, as the present paper reports as an ongoing research, it is encouraging enough to continue the elaboration and analysis. The reduced number of simulated conflicts should be related to the more strictly observance of the merging lane rules in the simulation when compared to the real world driver behaviour. That result shows as a more aggressive driver behaviour as to be set in the microsimulation with the aim to increase the number of conflicts. As far as the conflict severity is concerned, other candidate parameters for microsimulation calibration and validation are the values of TTC and speed that may be used as comparison between simulated and real world conflicts in the calibration process. In the VISSIM lane changing behaviour, driver aggressiveness can be controlled by modifying the maximum and accepted deceleration rates as well as the reduction rate of the deceleration value as the vehicle approaches its merge point [16]. Modifications to the maximum deceleration rates for the merging and trailing vehicles and car following headways may be conducted to determine the effect on number and severity of traffic conflicts.

\section{Conclusion}

The paper reports in progress research work to find a new approach to validate microsimulation models of $2+1$ sections, calibrated on observed data related both to traffic operation and observed conflicts. Microsimulations as well as the video survey were performed on typical Polish 2+1 segments characterized by short sections that characterizes such solutions from the traditional $2+1$ long section. Video were analyzed frame by frame at a $25 \mathrm{~Hz}$ frequency, vehicle trajectories were identified by using a software for image analysis able to correct distortion of images, by imputing real measures of fixed points in the frames. A part of the software precision, data filtering and control check of the results were applied to avoid false positive in conflict detection and TTC evaluation. Microsimulation models previously calibrated using traffic operation parameters were used to create virtual vehicle trajectories analyzed by SSAM to identify conflicts in the simulation environment. Comparison of simulated and observed conflicts, even at the early stage of investigation of the present research work, shown a significant linear correlation, but with a factor of $1 / 10$ between simulated and actual number of conflicts. That discrepancy in the total counts of conflicts is not necessary a problem because the sensitivity of number and severity of conflicts to changes in traffic condition and geometric characteristics of the $2+1$ section is mainly of interest in the calibration microsimulation for analysis of safety performances of alternative design scenarios (for example length of passing lane [15]).

The authors presented the results of the research project entitled "The effectiveness of the $2+1$ lane section, with particular focus on different solutions separating traffic directions" (DZP/RID-I55/10/2016) supported by the National Centre for Research and Development and the General Director for National Roads and Motorways.

\section{References}

1. S. Cafiso, C. D'Agostino, Assessing the stochastic variability of the Benefit-Cost ratio in roadway safety management, Accid. Anal. Prev. 93 pp. 189-197 (2016). doi:10.1016/j.aap.2016.04.027

2. S. Cafiso, C. D’Agostino, Reliability-Based Assessment of Benefits in Roadway 
Safety Management, Transp. Res. Rec. J. Transp. Res. Board. 2513 pp. 1-10 (2015). doi: $10.3141 / 2513-01$

3. C. D'Agostino, Investigating Transferability and Goodness of Fit of Two Different Approaches of Segmentation And Model form for estimating safety performance of

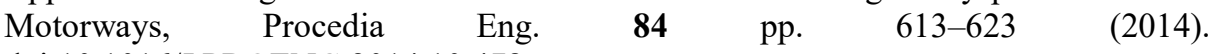
doi:10.1016/J.PROENG.2014.10.478

4. Use and application of microsimulation traffic models. AP-R286/06 (Austroads, 2006)

5. R. Dowling, A. Skabardonis, V. Alexiadis, Traffic Analysis Toolbox Volume III: Guidelines for Applying Traffic Microsimulation Software, FHWA-HRT-04-040 (Federal Highway Administration U.S. Department of Transportation, 2004)

6. F. Huang, P. Liu, H. Yu, W. Wang, Identifying if VISSIM simulation model and SSAM provide reasonable estimates for field measured traffic conflicts at signalized intersections, Accid. Anal. Prev. 50 pp. 1014-1024 (2013). doi:10.1016/J.AAP.2012.08.018

7. L. Pu, J. Rahul, Surrogate Safety Assessment Model (SSAM): Software User Manual. FHWA-HRT-08-050 (Federal Highway Administration, U.S. Department of Transportation, 2008)

8. C. Wang, N. Stamatiadis, Surrogate Safety Measure for Simulation-Based Conflict Study, Transp. Res. Rec. J. Transp. Res. Board. 2386 pp. 72-80 (2013). doi:10.3141/2386-09

9. R. Fan, W. Wang, P. Liu, H. Yu, Using VISSIM simulation model and Surrogate Safety Assessment Model for estimating field measured traffic conflicts at freeway merge areas, IET Intell. Transp. Syst. 7 pp. 68-77 (2013). doi:10.1049/ietits.2011.0232

10. U.E. Shahdah, F.F. Saccomanno, B. Persaud, Integrating Observational and Traffic Simulation Models for Priority Ranking of Unsafe Intersections, Transp. Res. Rec. J. Transp. Res. Board. 2280 pp. 118-126 (2012). doi:10.3141/2280-13

11. S. Cafiso, C. D’Agostino, R. Bąk, The Assessment of Road Safety for Passing Relief Lanes Using Microsimulation and Traffic Conflict Analysis, RSS2015 Spec. Issue Adv. Transp. Stud. 2 pp. 55-64 (2015)

12. D. Gettman, L. Pu, T. Sayed, S. Shelby, Surrogate Safety Assessment Model and Validation. Final Report. FHWA-HRT-08-051 (Federal Highway Administration, U.S. Department of Transportation, 2008)

13. M. Brewer, S. Venglar, K. Fitzpatrick, L. Ding, B. Park, Super 2 Highways in Texas. Operational and Safety Characteristics, Transp. Res. Rec. 2301 pp. $46-54$ (2012)

14. P. Kirby, G. Koorey, B. Wilmshurst, Operating characteristics and economic evaluation of 2+1 lanes with or without intelligent transport systems assisted merging. Research report 549 (NZ Transport Agency, 2014)

15. S. Cafiso, C. D’Agostino, M. Kieć, R. Bak, Safety assessment of passing relief lanes using microsimulation-based conflicts analysis, Accid. Anal. Prev. 116 pp. 94-102 (2018). doi:10.1016/j.aap.2017.07.001

16. PTV AG, User Manual VISSIM 5.10, (2008)

17. C.H. Spiegelman, E.S. Park, L.R. Rilett, Transportation Statistics and Microsimulation (CRC Press, Taylor \& Francis Group, Boca Raton, 2011)

18. S. Cafiso, C. D'Agostino, M. Kiec, Investigating the influence of passing relief lane sections on safety and traffic performance, J. Transp. Heal. 7 pp. 38-47 (2017). doi:10.1016/j.jth.2017.04.012 
19. S. Cafiso, C. D’Agostino, B. Persaud, Investigating the influence on safety of retrofitting Italian motorways with barriers meeting a new EU standard, Traffic Inj. Prev. 18 pp. 324-329 (2017). doi:10.1080/15389588.2016.1203424

20. Sputnik user manual (Vincenzo Pasquale Giofrè, Università della Calabria, 2011)

21. S. Cafiso, C. D’Agostino, Safety Performance Function for Motorways using Generalized Estimation Equations, Procedia - Soc. Behav. Sci. 53 pp. 900-909 (2012). doi:10.1016/J.SBSPRO.2012.09.939

22. E. Hauer, Observational Before-After Studies in Road Safety (Pergamon Press, Elsevier Science Ltd., Oxford, England, 1997) 